\title{
Dynamic Replacement of Virtual Service Resources Based on Tree Topology for Mobile Users in Virtual Networks
}

\author{
Sakie Horiuchi*, Takuji Tachibana \\ Graduate School of Engineering, University of Fukui, Bunkyo 3-9-1, Fukui-shi, Fukui, 910-8507 Japan. \\ * Corresponding author. Tel.:+81 77627 9971; email: sakie-h@network.fuis.u-fukui.ac.jp \\ Manuscript submitted June 25, 2018; accepted September 12, 2018. \\ doi: $10.17706 /$ jcp.13.12.1335-1348
}

\begin{abstract}
With the network virtualization technology, multiple virtual networks can be constructed on a substrate network with a part of substrate network resources. By using virtual service resources, a particular service can be provided to users over each virtual network. The quality of the service is affected by the amount of traffic on each virtual network. Therefore, virtual service resources are expected to be replaced in the virtual network based on the traffic change and topology change. This paper proposes a replacement method of virtual service resources for a virtual network with tree structure. In our proposed method, when the amount of traffic changes on any virtual node or the network topology changes, virtual service resources are replaced based on the tree structure so that the amount of traffic in the virtual network can be reduced. Moreover, we extend our proposed method so as to replace $2^{n}$ virtual service resources. We evaluate the performance of our proposed method with simulation. In numerical examples, it is shown that our proposed method can construct a larger number of virtual networks and maintain the quality of the service.
\end{abstract}

Key words: Virtual network, virtual service resource, traffic change, mobile user, tree topology.

\section{Introduction}

Network virtualization technology has been utilized actively worldwide [1]-[3]. By using the network virtualization technology, virtual networks are constructed on a substrate network with network resources such as CPU, memory, and bandwidth [4]-[7]. Virtual networks have already been used in some use cases [8], [9]. In the substrate network, each virtual network can be constructed and utilized independently each other. Even in the 5th generation networks, virtual networks are also expected to be constructed and utilized with network slicing technology [10]. In order to construct virtual networks, some construction methods have been proposed for substrate networks [11].

Over each virtual network, several kinds of services can be provided with users. Here, for a particular service, a server that is called virtual service resource is used in a virtual network. A service provider can provide its own service with users over the virtual network by utilizing the virtual service resource. The quality of the service is affected by the amount of traffic on each virtual network. When the amount of traffic is large (small) in the virtual network, the quality of provided service is low (high). In addition, if new nodes are added into or existing nodes are removed from the virtual network, the quality of provided service changes more significantly. Therefore, in order to maintain the quality of provided service, virtual service resource is expected to be replaced in the virtual network based on the traffic change and topology 
change.

With the spread of mobile terminals, virtual networks are also utilized by mobile users. In such a virtual network, the amount of traffic and the network topology may be changed dynamically if some users move frequently from an access point to another access point. Therefore, virtual service resources have to be replaced dynamically depending on the amount of traffic and the network topology. However, the detail of dynamic replacement of virtual network resources has not been considered yet.

In this paper, we propose a dynamic replacement method of virtual service resources for mobile users in virtual networks. In our proposed method, virtual service resources are replaced based on the tree structure so that the amount of traffic in the virtual network can be reduced. At first, we propose a dynamic replacement method for only a virtual service resource in the virtual network with tree structure. Note that this method has already been proposed in [12], but its performance evaluation is inadequate. Next, we propose a topology design method with spanning tree algorithm for our proposed dynamic replacement method. By using this method, any network topology can be changed into tree structure, and our proposed dynamic replacement method can be used in any topology. Moreover, we extend our proposed dynamic replacement method so as to replace $2^{n}$ virtual service resources. With those methods, it is expected that the quality of provided service can be maintained for mobile users. We evaluate the performance of our proposed methods with simulation and investigate the effectiveness of the proposed methods.

The rest of this paper is organized as follows. Section 2 introduces related work. Section 3 explains the detail of our three proposed methods. By using the three proposed methods, virtual service resources can be replaced for two cases in terms of the number of virtual service resources. Section 4 shows some numerical examples and Section 5 denotes conclusions.

\section{Existing Resource Allocation and Replacement of Virtual Network Resources for Mobile Users}

In virtual networks, for satisfying the quality of provided service, both a dynamic resource allocation and a dynamic replacement of virtual network resources have been proposed [13].

Now, we consider a virtual network with $N$ nodes. In this network, the $i$ th node is denoted as $n_{i}(\mathrm{i}=$ $1, \cdots N$ ) and the amount of resources of node $n_{i}$ is denoted as $m_{i}$. Moreover, $l_{i j}$ is a link between node $n_{i}$ and $n_{j}$, and the amount of resources of link $l_{i j}$ is denoted as $m_{i j}$. This method includes 1) dynamic resource allocation in a case where the amount of traffic changes in a virtual network and 2) dynamic replacement of virtual network resources, respectively.

When the amount of traffic for node $n_{i}$ changes from $\gamma_{i}$ to $\gamma_{i}{ }^{\prime}$, by using the dynamic resource allocation, the amount $m_{i}$ of resources for node $n_{i}$ is changed into

$$
m_{i}^{\prime}=\frac{m_{i} \gamma_{i}^{\prime}}{\gamma_{i}}
$$

As a result, the amount of resources for a node increases if the amount of traffic becomes large for the node. Moreover, when the amount of traffic for link $l_{i j}$ changes from $\gamma_{i j}$ to $\gamma_{i j}{ }^{\prime}$, by using this method, the amount $m_{i j}$ of resources for link $l_{i j}$ is changed into

$$
m_{i j}^{\prime}=\frac{m_{i j} \gamma_{i j}{ }^{\prime}}{\gamma_{i j}}
$$

As is the case with the node resources, the amount of link resources increases if the amount of traffic becomes large.

On the other hand, when a new node $n_{k}$ is added in to a virtual network, a new topology is designed by 
using shortest path betweenness. However, with the redesign of network topology, the quality of provided services cannot be maintained. Therefore, a virtual service resource is also replaced by using the dynamic replacement of virtual network services according to the following procedure.

i) Derive both the shortest path and the number of hops from the new added node $n_{k}$ to the node $n_{c}$ where a virtual service resource has been placed. Then, go to ii).

ii) Along the shortest path between $n_{c}$ and $n_{k}$, the virtual service resource moves to the adjacent node $n_{a}$ towards $n_{k}$ by one hop. Then, $n_{a}$ is set to $n_{c}$ and go to iii).

iii) If the quality of provided service can be satisfied, this algorithm finishes. If the quality is not satisfied and the number of hops between $n_{c}$ and $n_{k}$ is larger than one, go to ii). Otherwise, the dynamic replacement of virtual network services fails.

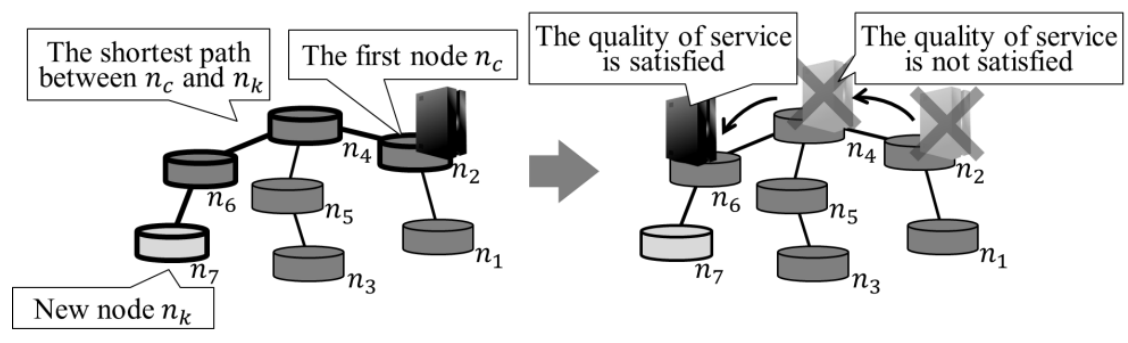

Fig. 1. Existing virtual service resource replacement where a virtual network service moves by one hop.

In this replacement method, the goal is to reduce the traffic on the route between the new node and the node where the virtual service resource is located (see Fig. 1). However, it is necessary to check whether the quality of the service is satisfied every time the placement is changed by one hop, and so it takes a long time to replace the virtual network service. Moreover, the replacement of virtual network service depends on both the new node and the node where a virtual service resource has been placed.

\section{Dynamic Replacement of Virtual Service Resources Based on Tree Topology}

In this section, at first, we propose a dynamic replacement of virtual service resources for a virtual network with tree topology. Next, in order to utilize the proposed replacement for every virtual network, we propose a topology design with spanning tree algorithm for virtual networks. By this topology design, even if a node is added into or removed from a virtual network with tree topology, the tree topology can be maintained. Moreover, we extend the proposed dynamic replacement method of a virtual service resource into the dynamic replacement method of $2^{n}$ virtual service resources.

\subsection{Dynamic Replacement of A Virtual Service Resource}

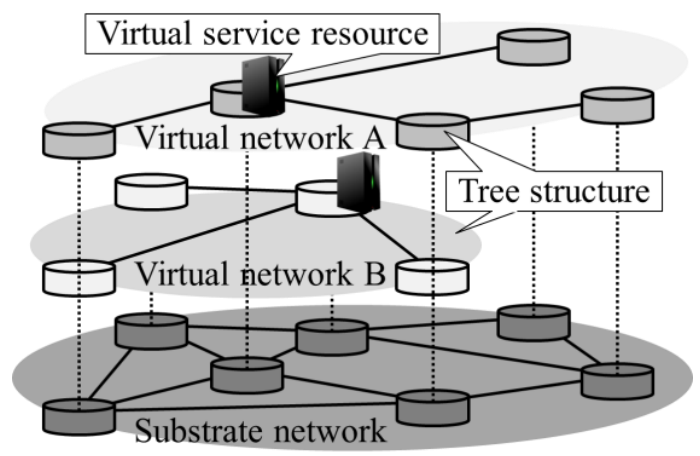

Fig. 2. Virtual service resource in virtual networks with tree structure. 
In this subsection, we propose a dynamic replacement method of a virtual network resource. By using this method, it is expected that users can utilize the virtual service resource appropriately.

Here, our proposed method can be utilized for a virtual service resource in a virtual network with tree structure (see Fig. 2). In the following, we consider a virtual network with tree structure where there are $N$ nodes. For the virtual network, a node whose degree is one is called leaf node. Users who connect to node $n_{i}$ utilize the virtual service resource and the total amount of traffic is denoted as $m_{i}$. In addition, the amount of traffic passing through node $n_{i}$ is denoted as $\gamma_{i}$.

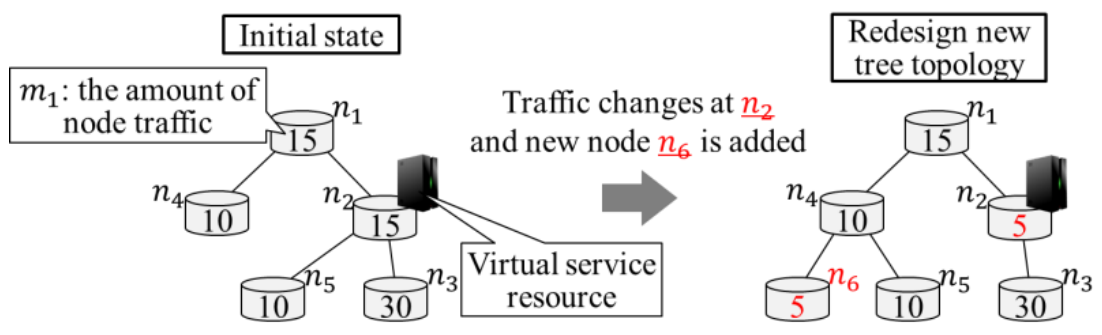

Fig. 3. An example of traffic change and topology change.

Now, we consider a case where the number of users changes or the network topology changes (see Fig. 3). In this case, the amount of traffic that passes through each node is changed, and hence the position of the virtual service resource is expected to be changed.

In our proposed dynamic replacement method, the virtual service resource is replaced dynamically according to the following steps.

i) Set $\gamma_{i}=m_{i}$ for each node $n_{i}$ and go to ii).

ii) Select the node $n_{p}$ whose $\gamma_{p}$ is the smallest among all leaf nodes, and go to iii).

iii) Set $\gamma_{q}=\gamma_{p}+\gamma_{q}$ for the only node $n_{q}$ that is connected to $n_{p}$. Next, the leaf node $n_{p}$ is removed from this process. After removing $n_{p}, n_{q}$ becomes one of the leaf nodes if the degree of $n_{q}$ is one. Then, go to iv).

iv) If the number of links is one in the virtual network, i.e., the number of nodes is two, this procedure finishes. Among the two nodes $n_{s}$ and $n_{t}$, a node $n_{s}$ whose amount traffic is larger is selected. Finally, the virtual service resource is placed in the selected node $n_{S}$. Otherwise, return to ii).

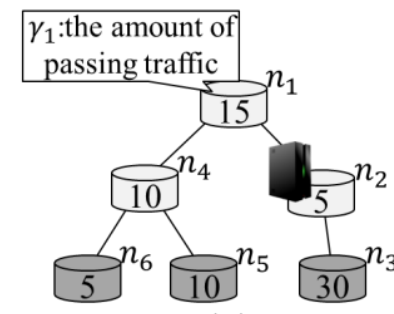

(a)

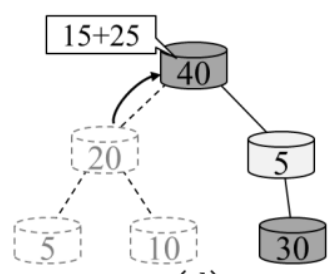

(d)

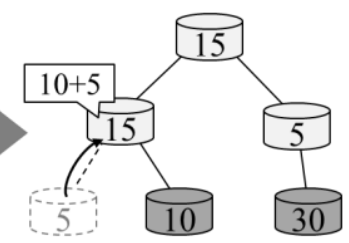

(b)

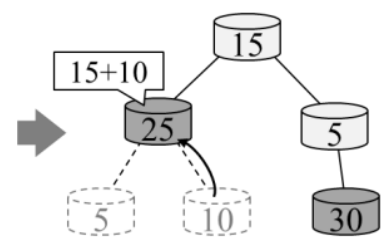

(c)

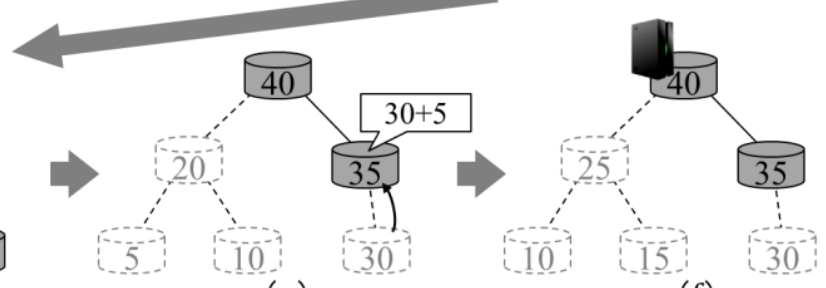

(e)

Fig. 4. An example of our proposed replacement of a virtual service resource. 
Fig. 4 shows an example of our proposed method for a virtual network where there are six nods. In this figure, the initial value of $\gamma_{i}(i=1, \cdots, 6)$ at node $n_{i}$ denotes the amount of traffic from users who are connected to $n_{i}$ at Step i) (see Fig. 4(a)). Among these six nodes, the leaf nodes are indicated in dark-gray. From three leaf nodes $n_{3}, n_{5}$, and $n_{6}$, at Step ii), $n_{6}$ with the smallest value of $\gamma_{i}$ is selected. Next, for $n_{4}$ that connects to $n_{6}, \gamma_{4}=\gamma_{4}+\gamma_{6}$ is calculated and $\gamma_{4}$ is set to 15 at Step iii) (see Fig. 4(b)). Then, $n_{6}$ is removed from this process. Note that $n_{6}$ is never removed from the virtual network. At Step iv). the number of links in the tree structure is larger than one, return to ii). Next, for $n_{5}$ whose $\gamma_{5}, \gamma_{5}$ is added to $\gamma_{4}$ (see Fig. 4(c)). The same process is repeated (see Fig. 4(d) and (e)), and two nodes $n_{1}$ and $n_{2}$ eventually remain. Finally, $n_{1}$ whose amount of traffic is larger than $n_{2}$ is selected, the virtual service resource is replaced in $n_{1}$ (see Fig. 4(f)).

\subsection{Topology Design with Spanning Tree Algorithm}

Our proposed dynamic replacement, that has explained in subsection 3.1, is available for only a virtual network with tree topology, and hence the proposed method can be utilized in a system where virtual networks with tree topology are constructed. On the other hand, the topology of the virtual network is changed when a new node is added into or an existing node is removed from a virtual network due to mobile user's movement. As a result, our proposed method may not be used even in a virtual network with tree structure when mobile users utilize the virtual service resource. Therefore, in our proposed topology design, a virtual network with tree structure is generated from the virtual network whose topology is not tree structure.

In the proposed method, the robustness of the substrate network is considered in order to maintain the quality of the service that has been provided in the substrate network. Here, we derive the Laplacian matrix $L$ from the amount of resources that have not been used in the virtual networks. Then, with the number $n$ of rows and columns of the Laplacian matrix, i.e., the number of nodes in the substrate node, the robustness of the substrate network is evaluated by

$$
\tau=2 n T_{r}\left(L^{+}\right),
$$

where $L^{+}$is the pseudo inverse of $L$ and $T_{r}$ is the sum of diagonal elements [11]. When the network robustness is high (low), $\tau$ is small (large). In the quality of the service, $\tau$ for the substrate network has to be smaller than the pre-defined threshold $\tau$. However, in the substrate network, the amount of network resources that have not been used in the virtual networks decreases when a new virtual network is constructed.

Therefore, the proposed topology design, a new tree topology is generated by excluding some links from the network topology. Here, for link $l_{i j}$ between $n_{i}$ and $n_{j}$ in the substrate network, the link cost $C_{i j}$ is given by

$$
C_{i j}=\tau+\omega_{i j} \frac{\partial \tau}{\partial \omega_{i j}}
$$

where $\omega_{i j}$ denotes the amount of link resources that have not been used in virtual networks. Note that this link cost shows the impact of $l_{i j}$ on the network robustness.

Our proposed method utilizes Kruskal's algorithm for driving the minimum spanning tree [14]. By designing the tree topology so as to minimize (4), the quality of the service that has been provided in the substrate network can be maintained. Here, Kruskal's algorithm can derive the minimum spanning tree faster and easier than Prim's MST algorithm.

In this method, a link is selected as target link in ascending order of the link cost $C_{i j}$. Then, the target link is added in the network topology if a cycle is not generated in the topology. By this topology design, a virtual network with tree structure can be generated from the virtual network with different topology. 


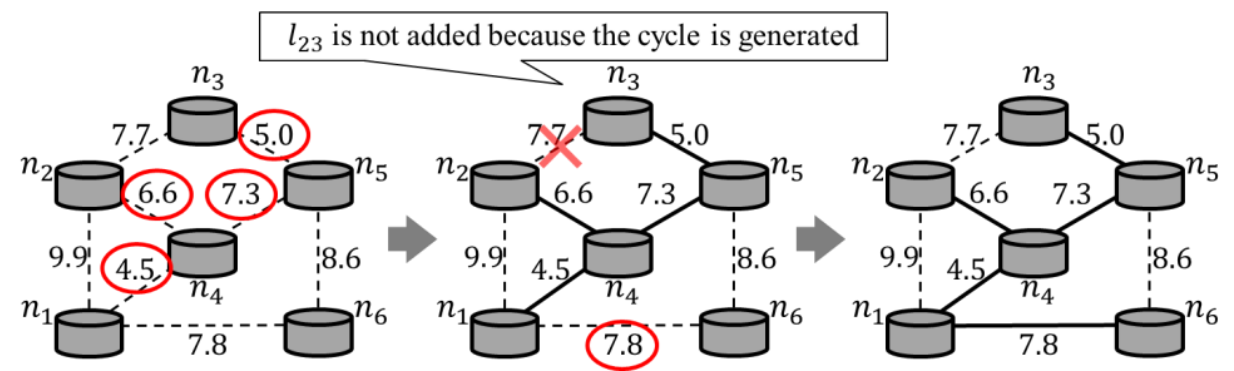

Fig. 5. An example of topology design with spanning tree algorithm.

Fig. 5 shows how a tree topology is generated with our proposed topology. In this figure, dot lines correspond to links in the original topology of a virtual network and the cost $C_{i j}$ is shown on each link. In our proposed design, eight links are arranged in ascending order of $C_{i j}$ such that $l_{14}, l_{35}, l_{24}, l_{45}, l_{23}, l_{16}, l_{56}$, and $l_{12}$. Moreover, in the initial state, there is no link in the network topology. In this example, links $l_{14}, l_{35}, l_{24}$, and $l_{45}$ in the ascending order are added because a cycle is not generated in the topology. However, when $l_{23}$ is added in the topology, a cycle is generated. Therefore, $l_{23}$ is not added and $l_{16}$, which does not generate a cycle, is added as the next link. Moreover, $l_{56}$ and $l_{12}$ are not added because a cycle is generated. Finally, a virtual network with tree topology is designed.

\subsection{Dynamic Replacement of $2^{n}$ Virtual Service Resources}

In subsection 3.1, we have proposed the replacement of one virtual service resource for a virtual network with tree structure. However, this method cannot be available for multiple virtual resources. In this subsection, we extend the method into a dynamic replacement for $2^{\mathrm{n}}$ virtual service resources.

In the extended dynamic replacement, in order to replace $2^{n}$ virtual service resources appropriately, the virtual network is divided into $2^{n}$ networks based on the amount of traffic. Then, a virtual service resource is placed in each network. By using this extended method, the total amount of traffic from users can be decreased. Note that we consider that each user utilizes the closest virtual network service in the virtual network.

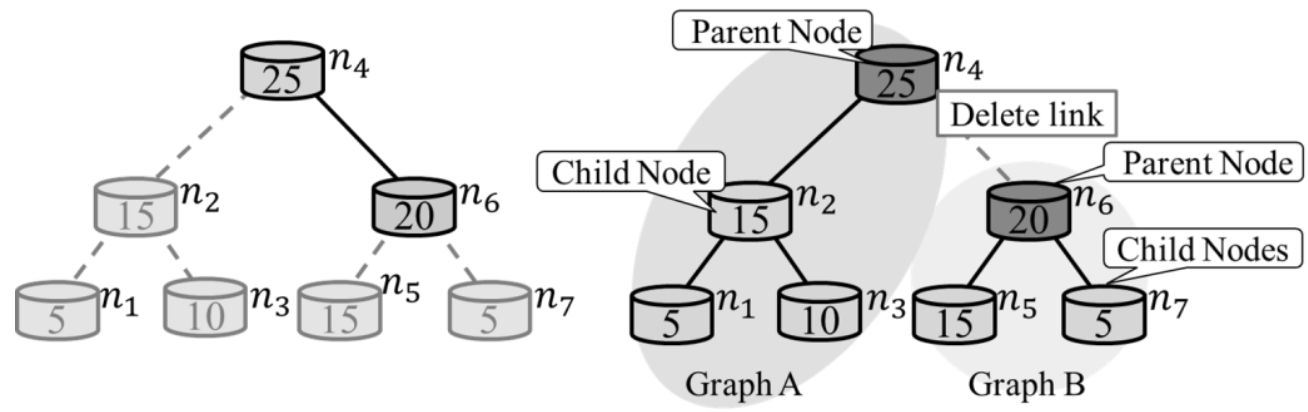

(a)

(b)

Fig. 6. Division of the virtual network into two networks.

In our extended method, at first, one link is selected by using the algorithm shown in subsection 3.1 (see Fig. 6(a)). Next, this link is removed and the original network is divided into two graphs (see Fig. 6(b)). Then, a virtual network resource is placed in each virtual network. Here, two virtual service resources are placed in two nodes that had been connected to the deleted link, respectively. Each node where the virtual service resource is denoted as the parent node $n_{q}$ (see Fig. 6(b)) and the position of virtual service resource is decided in each network according to the following algorithm. Fig. 7 also shows a flowchart of 
our virtual service resource replacement.

i) If the parent node $n_{q}$ has no child node, go to Step viii). if $n_{q}$ has only one child node, go to Step ii). If $n_{q}$ has multiple child nodes, go to Step v).

ii) Set $\gamma_{s}^{\text {new }}$ is set to $\gamma_{s}^{\text {new }}+m_{q}$ on the link $l_{s q}$ between the parent node $n_{q}$ and the child node $n_{s}$. Then, go to Step iii).

iii) In terms of $\gamma_{s}$ for the child node $n_{s}$, go to Step viii) if $\gamma_{s} \leq \gamma_{s}^{\text {new }}$ is satisfied. Otherwise, go to Step iv).

iv) Delete link $l_{s q}$, and the virtual network resource is replaced to the child node $n_{s}$. Then, $n_{s}$ is set to $n_{q}$ and return to Step i).

v) For the node $n_{t}$ with the highest traffic $\gamma_{t}$ on the child nodes, $\gamma_{t}^{\text {new }}=\gamma_{q}^{\text {new }}+\gamma_{q}-\gamma_{t}$ is derived. Then, go to Step vi).

vi) If $\gamma_{t} \leq \gamma_{t}^{\text {new }}$ is satisfied, go to Step viii). Otherwise, set $n_{t}$ to $n_{q}$ and return to Step i).

vii) Delete $l_{s t}$, set $n_{t}$ to $n_{q}$ and return to i).

viii) A virtual service resource is placed on the parent node $n_{q}$.

With these processes, two virtual network resources can be replaced in the virtual network, were a virtual network service is placed in a divided network. By using these processes again to each divided network, four virtual service resources in the virtual network. Finally, the processes are repeated $n$ times, and $2^{n}$ virtual service resources can be replaced.

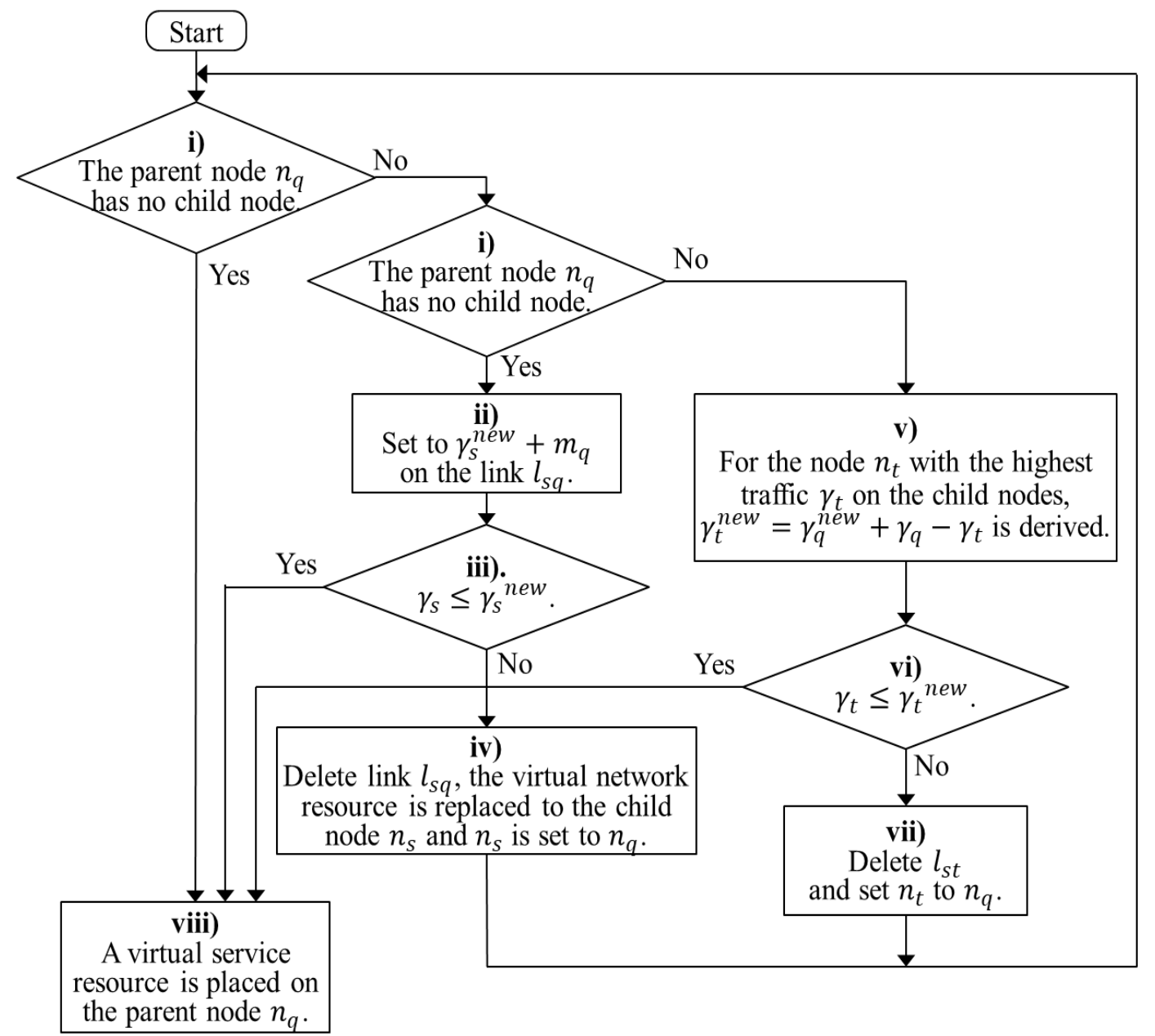

Fig. 7. Flowchart of extended virtual service resource placement. 


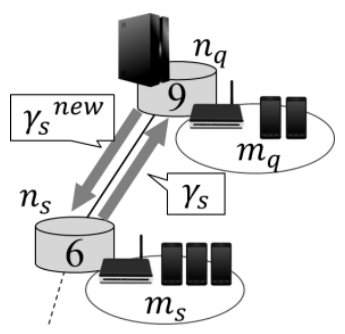

(a) A case of one child node.

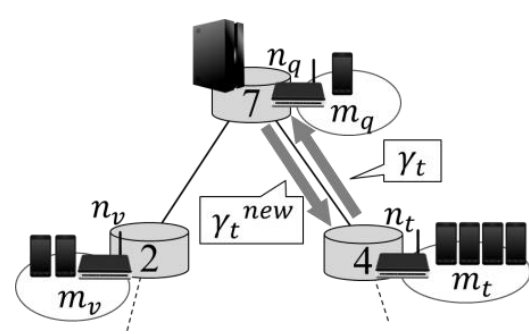

(b) A case of two child nodes.

Fig. 8. Replacement of multiple virtual service resources.

Fig. 8 (a) shows an example of Step iii) for the above algorithm. In this figure, the traffic on the link $l_{q s}$ between $n_{q}$ and $n_{s}$ is determined by comparing $\gamma_{s}$ with $\gamma_{s}^{\text {new }}$. On the other hand, Fig. 8 (b) shows an example of Step vi) for the above algorithm, where $n_{t}$ is the largest $\gamma_{t}$ among the child nodes of $n_{q}$. The traffic on the link $l_{q t}$ between $n_{q}$ and $n_{t}$ is determined by comparing $\gamma_{t}$ with $\gamma_{t}^{\text {new }}$. In both cases, the virtual service resource is replaced to the child node $n_{s}$ or $n_{t}$ from the parent node $n_{q}$ if the amount of traffic can be reduced.

\section{Numerical Examples}

In this section, we evaluate the performance of our proposed method with simulation. In Section 4.1, we investigate the impact of our proposed method for a virtual service resource. Next, in Section 4.2, we investigate the impact of our proposed method for multiple virtual service resources.

\subsection{Effectiveness of Our Proposed Method for A Virtual Service Resource}

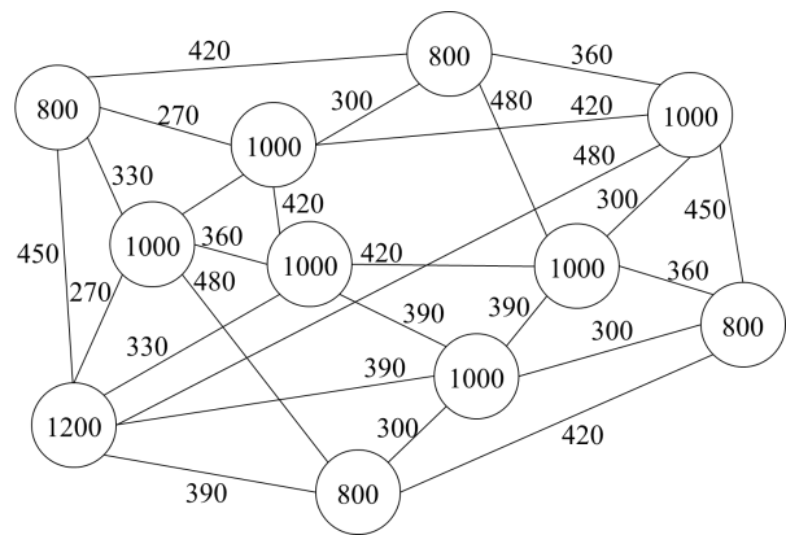

Fig. 9. Network topology.

In this subsection, we evaluate the performance of our proposed method in a substrate network shown in Fig. 9. In this substrate network, the amount of resources is from 800 to 1000 for each node and the amount of resources is from 270 to 480 for each link. For this substrate network, we assume that a new request for a virtual network construction arrives according to a Poisson process with rate 1.0. This request includes the information on $H$ nodes $(3 \leq H \leq 5)$ and the amount of resources that are needed for the constructed virtual network. The number $u_{i}$ of users for node $n_{i}$ is determined at random among $[30,100]$ and the amount of requested resources for $n_{i}$ depends on $u_{i}$. Upon receiving a new request of virtual network, a virtual network is constructed based on the robustness of the physical network [11]. When a new virtual network is constructed or reconstructed, it is checked whether the virtual network satisfies the quality of the service. Here, it is assumed that the node $n_{i}$ is connected with the virtual service resource $S_{i}$ and $u_{i}$ 
users utilize $S_{i}$ along the shortest path. The amount $\gamma_{i}$ of traffic for node $n_{i}$ is given by $\gamma_{i}=\alpha u_{i}$, where $\alpha$ is traffic coefficient, and the amount $\gamma_{p q}$ of traffic for link $l_{p q}$ is calculated from $\gamma_{i}$.

If all the amount $m_{p q}$ of resource for link $l_{p q}$ in the virtual network satisfy the following inequality (5), the quality of the service can be satisfied in this virtual network.

$$
\frac{\gamma_{p q}}{m_{p q}} \leq c
$$

As $c$ is larger, the amount of traffic that can be accommodated becomes large. If all of the following construction conditions are satisfied, a virtual network is constructed.

i) The robustness of the virtual network is smaller than the threshold (see [11] for more information).

ii) The amount of resources for each node and link is smaller than the amount of resources that can be provided by the substrate network.

iii) The amount of resource for every link in the virtual network satisfies (5).

Otherwise, the virtual network is not constructed and the request is rejected.

After constructing each virtual network, the amount of traffic changes according to the exponential distribution with rate $\lambda$. In this case, only the amount of traffic changes with a probability of 0.5 and a new node is added into the virtual network with a probability of 0.5 . When the amount of traffic changes, 40 users move between two nodes that are randomly selected among all nodes where the number of users is larger than 40. On the other hand, when a new node is added, the number of users for the added node is determined at random among $[30,100]$. When a new node is added, the topology is redesigned so that the construction condition is satisfied and the virtual service resource is replaced by our proposed method. We assume that the utilization time of the virtual network follows an exponential distribution with rate 1.0.

In this situation, we evaluate the effectiveness of our proposed method with simulation. In this case, the initial placement of a virtual network resource is performed at random, which is denoted as Proposed+Random. We also evaluate the performance of the conventional method [13] for the performance comparison. In addition, we evaluate the performance of the method where the initial placement of a virtual service resource is also determined with our proposed method, which is denoted as Proposed +3.1 . Here, traffic coefficient $\alpha$ is equal to 1.0 and $c$ is equal to 1.0 .

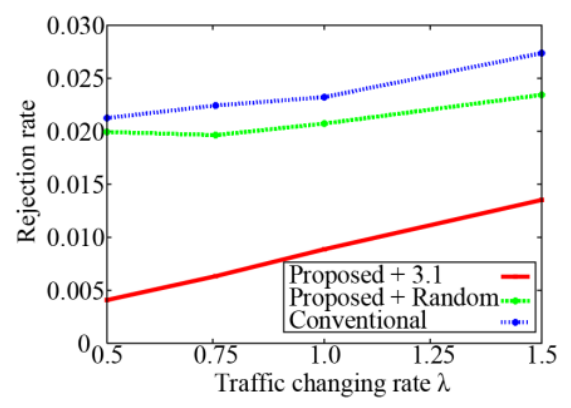

Fig. 10. Rejection rate vs. traffic changing rate $\lambda$.

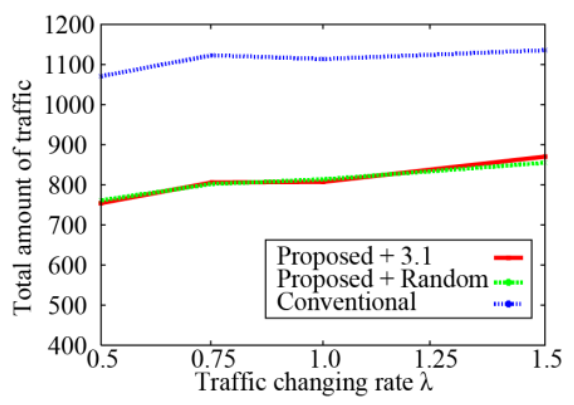

Fig. 11. Total amount of traffic vs. traffic changing rate $\lambda$.

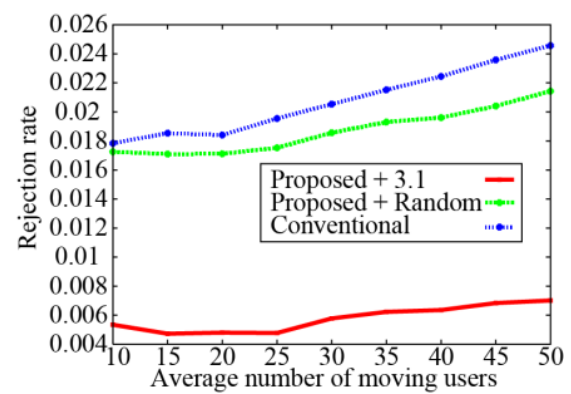

Fig. 12. Rejection rate vs. average number of moving users.

Fig. 10 shows the rejection rate of virtual network against the traffic changing rate $\lambda$ for network topology shown in Fig. 9. From this figure, we can find that the rejection rate for our proposed method is smaller than that for the conventional method regardless of the traffic changing rate $\lambda$. We can also find that the rejection rate becomes smaller by determining initial placement of a virtual service resource with our proposed method. Hence, by using our proposed method, a larger number of virtual networks can be constructed over a substrate network regardless of the traffic changing rate $\lambda$. Fig. 11 shows the total 
amount of traffic against the traffic changing rate $\lambda$. In this figure, we can find that the total amount of traffic for the three methods increases as the traffic changing rate $\lambda$ increases. However, we can find that the amount of traffic for our proposed method is smaller than that for the conventional method and that for the three methods is satisfied (5) regardless of the traffic changing rate $\lambda$. Therefore, the quality of the service can be satisfied in these virtual networks. Fig. 12 shows the rejection rate of virtual network against the average number of moving users. Here, the traffic changing rate $\lambda$ is equal to 1.5 . In this figure, we can find that the rejection rate for the three methods increases as the average number of moving users increases. However, we can find that the rejection rate for our proposed method is smaller than that for the conventional method regardless of the average number of moving users. This figure also shows that the rejection rate becomes smaller by determining initial placement of a virtual service resource with our proposed method.

From these results, our proposed method can construct a larger number of virtual networks while keeping the quality of the service. In addition, a larger number of virtual networks can be constructed over a substrate network by determining the initial placement of a virtual service resource with our proposed method. Therefore, in order to construct many virtual networks based on the amount of traffic, our proposed method is more effective than the conventional method.

\subsection{Effectiveness of Our Proposed Method for Multiple Virtual Service Resources}

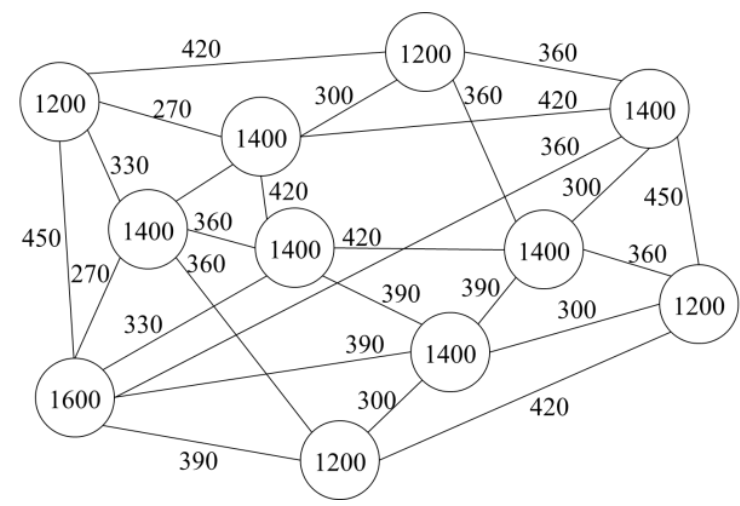

Fig. 13. Network topology.

In this subsection, we evaluate the performance of our proposed method in a substrate network shown in Fig. 13. In this substrate network, the amount of resources is from 1200 to 1600 for each node and the amount of resources is from 270 to 480 for each link. A new request for a virtual network construction arrives according to Poisson process with rate $\lambda_{1}$. This request includes the information on $H$ nodes ( $6 \leq H \leq 8)$ and the amount of resources that are needed for the constructed virtual network. The number $u_{i}$ of users for node $n_{i}$ is determined at random among [30,100] and the amount of requested resources for $n_{i}$ depends on $u_{i}$. In each virtual network, two virtual network resources are utilized. Each user utilizes the closest virtual network resources along the shortest path. After constructing each virtual network, the amount of traffic changes according to the exponential distribution with rate $\lambda_{2}$. In this case, only the amount of traffic changes with a probability of 0.5 and a new node is added into the virtual network with a probability of 0.5 . When the amount of traffic changes, 70 users move between two nodes that are randomly selected among all nodes where the number of users is larger than 70 . We also assume that the utilization time of the virtual network follows an exponential distribution with rate 1.0. For other parameters, we have used the same values as Section 4.1.

In this situation, we evaluate the effectiveness of our proposed method with simulation. In this case, the 
initial placement of a virtual network resource is performed with our proposed method in subsection 3.1, which is denoted as Proposed+3.1. We also evaluate the performance of the random method where two virtual network resources are placed at random, which is denoted as Random. In addition, we evaluate the minimum traffic allocation method for the performance comparison, that is denoted as Minimum traffic. In this method, two virtual network resources are placed by using full search so as to minimize the total amount of traffic.

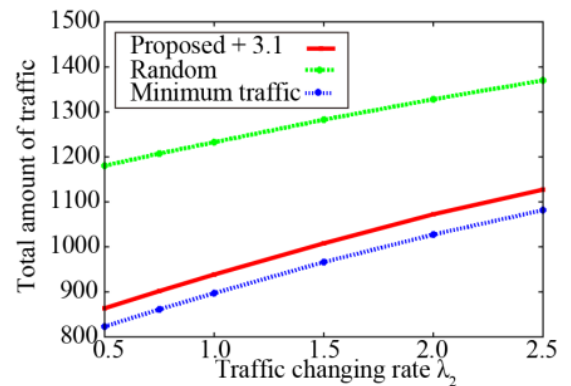

Fig. 14. Total amount of traffic vs. traffic changing rate $\lambda_{2}$.

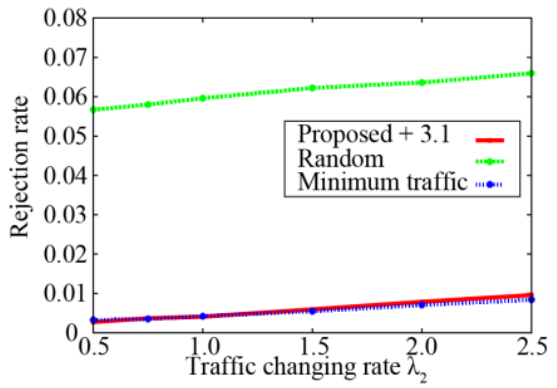

Fig. 15. Rejection rate vs. traffic changing rate $\lambda_{2}$.

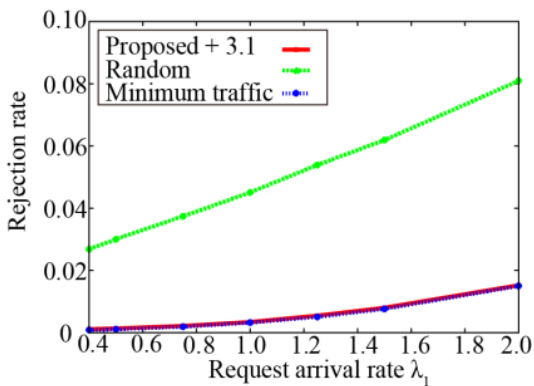

Fig. 16. Rejection rate vs. request arrival rate $\lambda_{1}$.

Fig. 14 shows the total amount of traffic in virtual network against the traffic changing rate $\lambda_{2}$. Here, the request arrival rate $\lambda_{1}$ is equal to 1.0. In this figure, we can find that the total amount of traffic for the three methods increases as the traffic changing rate $\lambda_{2}$ increases. However, we can find that the rejection rate for our proposed method is smaller than that for the random method regardless of the traffic changing rate $\lambda_{2}$. Fig. 15 shows the rejection rate of virtual network against the traffic changing rate $\lambda_{2}$. Here, the request arrival rate $\lambda_{1}$ is equal to 1.0. From this figure, we can find that the rejection rate for our proposed method is almost the same as that for the minimum traffic method. Therefore, our proposed method can construct a larger number of virtual networks regardless of the traffic changing rate $\lambda_{2}$. Fig. 16 shows the rejection rate of virtual network against the request arrival rate $\lambda_{1}$. Here, traffic changing rate $\lambda_{2}$ is equal to 1.0. In this figure, we can find that the rejection rate for the three methods increases as the request arrival rate $\lambda_{1}$ increases. From this figure, we can also find that the rejection rate for our proposed method is almost the same as that for the minimum traffic method. Therefore, our proposed method can construct a larger number of virtual networks regardless of the request arrival rate $\lambda_{1}$.

From these results, our proposed method can construct a larger number of virtual networks while keeping the quality of the service. Therefore, in order to construct many virtual networks based on the amount of traffic, our proposed method is almost the same as the minimum traffic method.

In order to investigate the impact of the number of virtual service resources, we evaluate the performance of our proposed method in a substrate network. In the substrate network, the number of nodes is 32 and the average degree of each node is 4.71. In addition, the amount of resources is 1000 for each node and the amount of resources is 500 for each link in the substrate network. A new request for a virtual network construction arrives according to Poisson process with rate 1.0. This request includes the information on $H$ nodes $(22 \leq H \leq 24)$ and the amount of resources that are needed for the constructed virtual network. The number $u_{i}$ of users for node $n_{i}$ is determined at random among $[30,100]$ and the amount of requested resources for $n_{i}$ depends on $u_{i}$. In each virtual network, $2^{n}$ virtual network resources are utilized. Each user utilizes the closest virtual network resources along the shortest path. After constructing each virtual network, the amount of traffic changes according to the exponential distribution with rate 1.0. In this case, only the amount of traffic changes with a probability of 0.5 and a new node is added into the virtual network with a probability of 0.5 . When the amount of traffic changes, 70 users move 
between two nodes that are randomly selected among all nodes where the number of users is larger than 70. We also assume that the utilization time of the virtual network follows an exponential distribution with rate 1.0. For other parameters, we have used the same values as Section 4.1.

In this situation, we evaluate the effectiveness of our proposed method in subsection 3.3 with simulation. In this case, the initial placement of a virtual network resource is performed with our proposed method in subsection 3.1, which is denoted as Proposed +3.1 . We also evaluate the performance of the random method where $2^{n}$ virtual network resources are placed at random, which is denoted as Random. In addition, we evaluate the minimum traffic allocation method for the performance comparison, that is denoted as Minimum traffic. In this method, $2^{n}$ virtual network resources are placed by using full search so as to minimize the total amount of traffic.

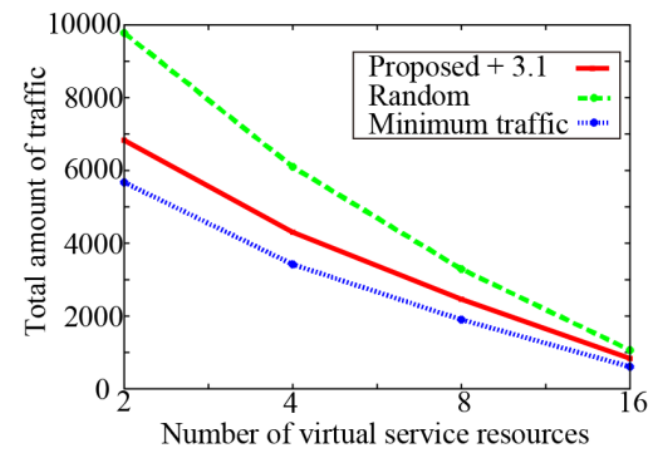

Fig. 17. Total amount of traffic vs. number of virtual service resources.

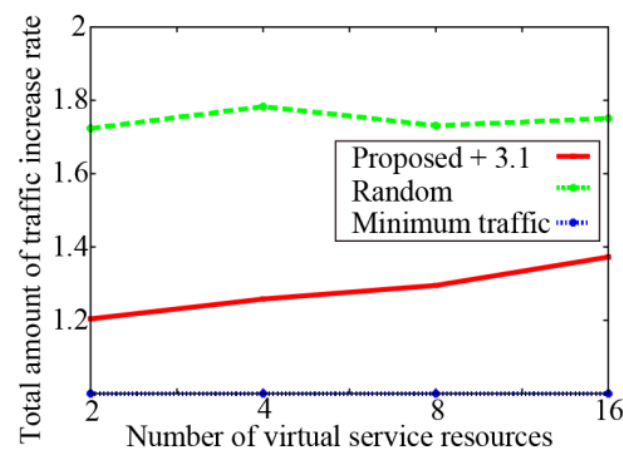

Fig. 18. Total amount of traffic increase rate vs. number of virtual service resources.

Fig. 17 shows the total amount of traffic in the virtual network against the number of virtual service resources. In this figure, we can find that the total amount of traffic for the three methods decreases as the number of virtual service resources increases. From this figure, we can also find that the total amount of traffic for our proposed method is smaller than that for the random method regardless of the number of virtual service resources. Fig. 18 shows the total amount of traffic increase rate against the number of virtual service resources. In this figure, note that the total amount of traffic for the minimum traffic method is taken as 1.0. From this figure, we can find that the amount of traffic increase rate for our proposed method is smaller than that for the random method regardless of the number of virtual service resources.

From these results, our proposed method can keep the quality of the service regardless of the number of virtual service resources.

\section{Conclusion and Future Work}

In this paper, in order to maintain the quality of provided service, we proposed a dynamic replacement method of virtual service resources for mobile users in virtual networks. This replacement method can decrease the total amount of traffic based on the tree structure of virtual network after the traffic change and the topology change. In addition, we proposed a topology design method with spanning tree algorithm for our proposed dynamic replacement method. Moreover, we extended our proposed dynamic replacement method so as to replace $2^{n}$ virtual service resources by dividing the original virtual network into $2^{n}$ graphs based on the amount of traffic. We evaluated the performance of our proposed method with simulation. From numerical examples, we found that our proposed method can decrease the amount of traffic and the quality of the service can be maintained even if the traffic and the topology change. From the performance comparison, we found that our proposed method can construct a larger number of virtual networks than the conventional method. In addition, we found that our proposed method can keep the 
quality of the service regardless of the number of virtual service resources. In our future work, we would like to evaluate the performance of our proposed method by demonstration experiment and plan to devise the method to replace $R\left(R \neq 2^{n}\right)$ virtual service resources.

\section{Acknowledgment}

We would like to thank Mr. Yuki Koyanagi for giving us his valuable comments and suggestions.

\section{References}

[1] GENI. Retrieved from http://www.geni.net/

[2] PlanetLab. Retrieved from http://www.planet-lab.org/

[3] Emulab-Network Emulation Testbed. Retrieved from http://www.emulab.net/

[4] Zhu, Y., \& Ammar, M. (2006). Algorithms for assigning substrate network resource to virtual network component. Proceedings of IEEE International Conference on Computer (pp. 1-12). Barcelona, Spain.

[5] Yu, M., Yi, Y., Rexford, J., \& Chiang, M. (2008). Rethinking virtual network embedding: Substrate support for path splitting and migration. ACM SIGCOMM Computer Communication Review, 38(2), 17-29.

[6] Dekker, A. H., \& Colbert, B. D. (2004). Network robustness and graph topology. Proceedings of Australasian Computer Science Conference (pp. 359-368). Dunedin, New Zealand.

[7] He, J., Zhang-Shen, R., Li, Y., Lee, C., Rexford, J., \& Chiang, M. (2008). DaVinci: Dynamically adaptive virtual networks for a customized internet. Proceedings of ACM Conference on Emerging Networking Experiment and Technology (pp. 1-12). Madrid, Spain.

[8] Casado, M., \& McKeown, N. (2005). The virtual network system. Proceedings of the 36th SIGCSE Technical Symposium on Computer Science Education (pp. 76-80). St. Louis, Missouri, USA.

[9] Iguchi, N. (2017). Virtual IP network practice system with software agent. Proceedings of International Conference on Complex, Intelligent, and Software Intensive System (pp. 711-720).

[10] Nakao, A., Du, P., Kiriha, Y., Granelli, F., Gebremariam, A. A., Taleb, T., \& Bagaa, M. (2017). End-to-End network slicing for 5G mobile networks. Journal of Information Processing, 25(1), 153-163.

[11] Mori, M., Tachibana, T., Hirata, K., \& Sugimoto, K. (2011). A proposed topology design and admission control approach for improved network robustness in network virtualization. Proceedings of IEEE Global Communications Conference (pp. 1-5). Texas, USA.

[12] Horiuchi, S., \& Tachibana, T. (2018). Replacement of virtual service resources for virtual networks with tree structure. Proceedings of International Conference on Computer and Electrical Engineering.

[13] Koyanagi, Y., \& Tachibana, T. (2014). Dynamic Resource Allocation Based on Amount of Traffic in Virtual Networks (Report Vol. 113, No. 473). Miyazaki, Japan: IEICE Technical Report.

[14] Kruskal, J. B. (1956). On the shortest spanning subtree of a graph and the traveling salesman problem. Proceedings of the American Mathematical Society (pp. 48-50).

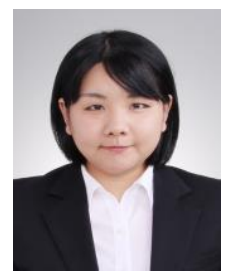

Sakie Horiuchi received the B. Eng. degree from the Faculty of Engineering, University of Fukui, Japan, in 2014. She received the M. Eng degree from Graduate School of Engineering, University of Fukui, Japan, in 2016. Since 2016, she has been a doctor course student at Graduate School of Engineering, University of Fukui, Japan.

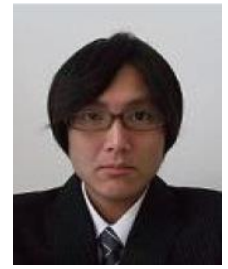

Takuji Tachibana received the B. Eng degree from the Department of Systems Engineering, Nagoya Institute of Technology, Japan, in 2000. He received the M. Eng. and Dr. Eng. degrees 
from the Department of Information Systems, Graduate School of Information Science, Nara Institute of Science and Technology, Japan, in 2001 and 2004, respectively. From 2004 to 2006, he was an expert researcher in the Information and Network Systems Department, National Institute of Information and Communications Technology, Japan. From 2006 to 2011, he was an assistant professor at the Department of Information Systems, Graduate School of Information Science, Nara Institute of Science and Technology, Japan. Since 2011, he has been an associate professor at Graduate School of Engineering, University of Fukui, Japan. His research interests include network virtualization, network architectures in optical networking, and performance analysis of computer and communication systems. He is a member of the IEEE, the Institute of Electronics, Information and Communication Engineers (IEICE), and the Operations Research Society of Japan. 